\title{
Philosophiques
}

\section{Peter Royle, The Sartre-Camus Controversy. A Literary and Philosophical Critique. Ottawa : University of Ottawa Press, 1982 (Collection Philosophica).}

\section{Walter Moser}

Volume 13, numéro 1, printemps 1986

URI : https://id.erudit.org/iderudit/203313ar

DOI : https://doi.org/10.7202/203313ar

Aller au sommaire du numéro

Éditeur(s)

Société de philosophie du Québec

\section{ISSN}

0316-2923 (imprimé)

1492-1391 (numérique)

Découvrir la revue

Citer ce compte rendu

Moser, W. (1986). Compte rendu de [Peter Royle, The Sartre-Camus Controversy. A Literary and Philosophical Critique. Ottawa : University of Ottawa Press, 1982 (Collection Philosophica).] Philosophiques, 13(1), 182-188.

https://doi.org/10.7202/203313ar d'utilisation que vous pouvez consulter en ligne.

https://apropos.erudit.org/fr/usagers/politique-dutilisation/ 
PETER ROYLE, The Sartre-Camus Controversy. A Literary and Philosophical Critique. Ottawa: University of Ottawa Press, 1982 (Collection Philosophica).

$$
\text { par Walter Moser }
$$

Voulez-vous présenter, comprendre et peut-être même arbitrer le conflit entre deux grands auteurs et penseurs? - Choisissez la méthode comparative. Mettez-les face à face, sur un terrain qu'ils ont en commun et faites ressortir ce qui les oppose. Braquez votre attention sur l'un et sur l'autre, à tour de rôle, tout en faisant la navette argumentative entre le fond commun et les différences. Engagez-vous dans le jeu logique de l'invariance et des variations. Et, si possible, profitez de votre position extérieure, et

4. A.H. ARMSTRong. The Cambridge History of Later Greek and Early Medieval Philosophy. Cambridge, University Press, 1967. [Armstrong est l'éditeur de ce collectif, dont il signe la troisième partie, consacrée à Plotin, aux pages 195-268.]

5. A.H. Armstrong. Plotinian and Christian Studies. London, Variorum, 1979; 384 pp. [Recueil de 24 études]

6. H.J. Blumenthal and R.A. Markus, Editors. Neoplatonism and Early christian Thought. Essays in honour of A.H. Armstrong. London, Varioru, 1981 ; X-251 pp. 
peut-être supérieure, de «troisième personne» (p. 92) pour couronner vos efforts d'une synthèse qui neutralisera différences et hostilités en réunissant vos deux adversaires sous un chapeau que vous leur aurez confectionné.

Ce procédé a fait ses preuves, et P.R. a bien fait de le suivre pour explorer ce qu'il appelle la controverse entre Sartre et Camus. Il part de la rupture qui s'annonce entre les deux auteurs français après la publication de L'bomme révolté. Il refuse cependant d'expliquer ce fait exclusivement sur le plan de leurs différences politiques. Il élargit la question et inclut dans son analyse des considérations sur les deux personnalités, mais surtout sur leur pensée philosophique. Voici donc l'objectif de son travail: «The object of this book is to bring out and to elucidate these philosophical differences, and to do so as far as possible through philosophical exegeses of works of literature» (p. 1).

Et le résultat? Eh bien, sa configuration est prévisible, puisqu'elle est donnée par le procédé adopté. L'auteur constate d'abord l'identité du terrain commun: "Sartre and Camus are involved with the same issues, and use many of the same terms and techniques» (p. 87), ensuite il marque les différences : "Sartre is an existential phenomenologist in the grand European philosophical tradition which starts with Descartes; Camus is a disabused heir of the Enlightenment» (p. 87), ce qui se reflète dans leur attitude à l'égard de la littérature: "Sartre lost interest in ethics, so he progressively abandoned art» et se convertit à l'action directe et à l'analyse marxiste (p. 90), tandis que Camus « retained intact a belief in the power of the word», c'est-à-dire il n'abandonne pas la création littéraire dans laquelle se réalise son imagination utopique (p. 88). Finalement il fait des affirmations visant à substituer, sous différentes formes, au «Sartre vs Camus» un «Sartre et Camus». La forme peut être celle de la complémentarité : «On philosophical questions Sartre is generally closer to the truth than Camus, whereas on practical political ones it is generally the reverse» (p. 92). Cette complémentarité prend la forme d'un chiasme si l'on affirme que Sartre est davantage philosophe mais moins humaniste, et Camus moins philosophe mais plus humaniste, surtout dans ses essais et œuvres littéraires. Finalement l'auteur n'hésite pas à donner la forme de l'oxymoron à cette synthèse : "If Sartre (...) is a romantic rationalist, Camus is a classical anti-rationalist ; and the oxymoron in the label points to equivalent tensions in each of them » (p. 87).

Voilà donc le projet et le résultat de ce livre. Décrire les développements qui relient ces deux moments extrêmes n'est pas chose facile, car l'auteur suit une argumentation doublement zigzaguante entre l'analyse philosophique et l'interprétation littéraire d'une part et entre les deux auteurs d'autre part.

Le premier chapitre est consacré à l'œuvre de Camus, L'bomme révolté, qui serait à l'origine du conflit avec Sartre. Il présente l'argument de Camus et en critique la nature peu logique et peu philosophique. L'auteur insiste 
sur les erreurs qui montrent le manque d'expérience et les malentendus de Camus philosophe.

L'Étranger, dans le deuxième chapitre, fait également l'objet d'une présentation très critique, plus élaborée cependant. Cette œuvre littéraire est traitée comme un roman à thèse; elle proposerait une représentation personnifiée de la notion philosophique de l'absurde. Meursault devait être le héros absurde, mais P.R. s'engage à prouver que toute représentation romanesque de l'homme absurde se solde par un échec, car - pour aller vite - tout acte d'écrire comporte toujours une production de sens et, par conséquent, la négation de l'absurde. L'auteur conclut: "the absurd is inviable literarily » (p. 22) et, plus généralement : «Camus's early philosophy is both literarily and philosophically inviable» (p. 23).

L'auteur semble donc endosser, même radicaliser la critique que Sartre a adressée au roman de Camus. On est currieux alors de connaître le sort qu'il réserve aux œuvres littéraires de Sartre. En fait, à travers le traitement qui lui est accordé dans le troisième chapitre, le roman La Nausée apparaît comme une cuvre plus intéressante et plus importante. Sartre aurait évité les pièges de la représentation littéraire (P.R. rappelle souvent que pour Sartre faire de la littérature, c'est tricher) dans lesquels aurait donné Camus. De nouveau, même s'il semble attribuer à la littérature une «valeur intrinsèque» (p. 28), l'auteur prend le roman comme un univers fictionnel où se trouvent mises à l'épreuve des notions philosophiques. Il lit donc $L a$ Nausée sur l'arrière-plan de L'Être et le néant. Il s'agit de trouver «la philosophie de La Nausée» (p. 27). Ou plus exactement, de vérifier l'adéquation des moyens de la représentation littéraire (formes narratives, style, personnages, thèmes) aux notions philosophiques que ces moyens sont censés véhiculer. Une analyse thématique du réseau des images récurrentes de La Nausée constitue peut-être le moment le plus intéressant de cette lecture, mais elle amène le critique à admettre que toutes ces images ne sont pas au service d'idées philosophiques et que certaines renvoient aux fantasmes personnels de leur auteur.

Imperceptiblement l'analyse glisse de l'examen de la manifestation littéraire des catégories philosophiques à ces dernières elles-mêmes. Malgré son préjugé favorable à l'égard de Sartre philosophe (selon P.R. le plus grand philosophe français de notre siècle), l'auteur lui adresse cette critique : " his vision of the world, which is a dark if not a pessimistic one, has contaminated his ontology and led him to false philosophical conclusions » (p. 35). Cependant, convaincu que, contrairement à L'Étranger, La Nausée est une réussite littéraire, il formule cette conclusion sur le mode interrogatif : «Does this mean that, paradoxically, success depends at least in part, on philosophical error?» (p. 40).

Le prochain texte retenu pour l'analyse est Le Mur, dans le chapitre quatre. P.R. qualifie ce récit de « histoire kantienne» (pp. 41 et 45), car elle pose, à l'instar de Kant, la question du mensonge utile. Cependant, Le Mur 
prend une tournure peu kantienne à la fin et sert plutôt à illustrer la doctrine sartrienne de la responsabilité totale de l'individu.

Suit un chapitre intitulé «Nature» qui porte sur plusieurs textes de Sartre, dont aucun n'est littéraire. Il s'agit donc d'un intermezzo philosophique qui met notre auteur aux prises avec Sartre philosophe. «Aux prises» est le terme approprié, car P.R. commence par élaborer une position très critique à l'égard de Sartre, en particulier quant à son traitement des concepts de «nature», «homme» et surtout «nature humaine» qui joue un rôle important chez Camus, mais est rejeté par Sartre.

Un véritable reproche est formulé à l'adresse de Sartre: d'être au fond un cartésien antihistorique (pp. 55-56), ce qui ne lui permettrait pas d'être à la hauteur de Hegel, Husserl, Heidegger auxquels il se réfère explicitement. Toutefois, après avoir montré ce que P.R. appelle les erreurs de Sartre («Why does Sartre go wrong?», p. 55), il les fait disparaître en réconciliant les éléments potentiellement contradictoires grâce à l'introduction de différents niveaux de rationalité (p. 54). L'intégrité philosophique de Sartre est ainsi sauvée et sa supériorité sur Camus philosophe définitivement établie, pendant que s'annonce déjà le chiasme de la synthèse finale: meilleur comme philosophe, Sartre doit céder le pas à Camus dans le domaine de la politique: "the political views he [Camus] held in the immediate post-war years seem to me to have been sounder on the whole than those of Sartre» (p. 51).

Le chapitre le plus long du livre est consacré à La Peste de Camus. Il s'agit à nouveau d'identifier les contenus philosophiques sous les formes littéraires. Cette interprétation accorde une grande place à une certaine rhétorique didactique. Question: quelle est la signification de la peste, de l'isolement de la ville d'Oran? Réponse, donnée après avoir testé et rejeté trois réponses fausses ou incomplètes: "Just as the close society of Oran is an image of all societies, including open ones, so, then, the situation depicted in The Plaque is an image of the human condition» (p. 71). Après avoir donné de la sorte dans l'interprétation allégorique, l'auteur cherche à identifier le message du roman: "The message of The Plaque isn't that we shouldn't remember and shouldn't hope, but that we shouldn't entertain illusory hopes based on apocalyptic conceptions of rosy, if distant, future.» (p. 70).

Finalement, il passe en revue tous les personnages de La Peste afin de vérifier avec quelle fidélité ils véhiculent les idées de leur créateur. Ceci mène à la question de la mauvaise foi de Camus auteur (pp. 84-85), question qui trouvera une réponse étrange : «Bad faith, then, may well be at the root of seriousness (...). Camus is in the position of the man who knows all his efforts are doomed to failure, yet believes he has a duty written in Heaven" (p. 85). La thèse du mauvais philosophe se confirme: la pensée de Camus n’a pas la rigueur nécessaire pour un philosophe, mais, à la fin, la fausseté 
des réponses philosophiques est rachetée par une forme artistiquement cohérente (p. 86).

La rupture entre Sartre et Camus est-elle pour autant expliquée? Certes le scénario - visible et caché - de cette brouille a été élargi et approfondi par rapport à la version simpliste qui réduit tout à l'antagonisme des positions politiques. P.R. a le mérite d'avoir opéré cet approfondissement en incluant dans son analyse les personnalités et les engagements pratiques de Camus et de Sartre. Mais surtout d'avoir exploré l'interaction difficile à décrire entre la philosophie et la littérature chez les deux auteurs.

Comme l'objectif explicite de son auteur l'indique, cette étude sur Sartre et Camus se situe dans la région frontalière où philosophie et littérature se séparent et se côtoient à la fois. Comment concevoir cette relation? - c'est là une des grandes questions sous-jacentes à ce livre. Conscient de sa portée, P.R. l'aborde explicitement dans son introduction, mais les réponses implicites qu'il fournit tout au long de ses analyses, sont tout aussi importantes. En réunissant les éléments pertinents, et en schématisant, voici ce qu'on trouve.

Si la philosophie est le domaine des idées, des concepts et de leur articulation qui vise toujours la vérité, la littérature, pour sa part, est plus difficile à cerner, peut-être à cause d'une polyvalence intrinsèque qui lui est attestée par le philosophe ( I believe that works of literature are polyvalent», p. 3). Contrairement à la philosophie qui a le souci de transmettre les pensées avec un maximum de clarté et de transparence, la nature esthétique de la représentation littéraire fait en sorte que ces mêmes pensées sont déformées, mystifiées. Serait-ce parce qu'elle transpose le général (les concepts, la vérité) dans le particulier (les situations, les personnages), ou parce que, en plus d'exprimer la pensée de l'auteur (ce qui semble être la fonction principale du texte littéraire), la littérature exprime aussi ses fantasmes? Ou encore à cause de son souci esthétique pour la cohérence interne de l'œuvre? Quoi qu'il en soit, l'usage qui est fait du langage en littérature inspire de la méfiance au philosophe.

Il est évident que P.R. aborde cette relation en philosophe (cf. son crédo de philosophe: «my object is truth», p. 4), non sans charger cette identité d'un certain pathétique: « as philosophers we have the right and the duty...» (p. 15). Il en découle un programme précis et univoque quand il aborde le texte littéraire: «My desire to extract from literature truths and falsehoods about man and his world» (p. 3). Faire une critique philosophique d'une œuvre littéraire veut donc dire retraduire le particulier en généralité, extraire concepts et vérité de leur enveloppe littéraire. Oserait-on dire: racheter les contenus philosophiques de leur chute dans une forme esthétique? - le ton presque moralisateur du philosophe qui décrit sa tâche fondamentale en face du texte littéraire semble justifier une telle formulation: «literature needs to be demystified» (p. 2). 
Le parcours méthodologique qui découle de ces présuppositions théoriques peut être reconstruit ainsi :

1) le philosophe étudie les œuvres philosophiques de Sartre et de Camus. Il connaît leur philosophie au point de pouvoir en articuler une critique et dire ce qui y est vrai et faux, bonne procédure et erreur.

2) il aborde les œuvres littéraires des mêmes auteurs pour en extraire le contenu philosophique et pour évaluer si la forme littéraire, et plus spécifiquement narrative, exprime adéquatement ce contenu.

3) les idées philosophiques ainsi récupérées et épurées sont réintroduites dans l'analyse philosophique.

Il va sans dire que, dans un tel procédé, la littérature ne constitue qu'un détour que ferait l'idée ou le concept philosophique dans une zone périlleuse. C'est à peine si l'auteur se pose des questions sur les raisons d'être de cette zone périlleuse du langage et sur sa fonction particulière chez Sartre et Camus.

Il est vrai que la critique philosophique de P.R. comporte des pages intéressantes; par exemple quand il analyse concrètement la forme narrative de L'Étranger pour évaluer si celle-ci peut rendre la notion philosophique de l'absurde (pp. 14-22). Mais il y a dans un tel procédé le danger de substantialiser une forme littéraire par rapport à une notion philosophique qu'elle serait, en principe, capable ou disqualifiée de manifester.

Un tel procédé présente aussi le désavantage de produire des critiques négatives, dans le sens qu'il a tendance à aboutir au constat d'échec de la mission philosophique que l'œuvre littéraire aurait eu la tâche d'accomplir. Ce type de critique donne des résultats particulièrement décevants dans le cas de L'Étranger qui, philosophiquement réussi ou non, a eu un grand impact auprès du public. Les lecteurs auraient-ils eu tort de lire une ouvre déclarée non-viable par la critique philosophique?

C'est dans un cas pareil que la critique pratiquée par P.R. me paraît particúlièrement improductive. Ce qui est en partie dû au fait que l'auteur s'est volontairement enfermé dans le circuit concept philosophique-forme littéraire-concept philosophique. Un coup d'œil jeté par-dessus la barrière, du côté de la critique littéraire - catégorie dont l'auteur reconnaît l'existence de principe (cf. p. 15) - aurait été profitable. Par exemple dans les travaux de Pierre V. Zima (L'Ambivalence romanesque, Paris: Le Sycomore, 1980, et L'Indifférence romanesque, Paris: Le Sycomore, 1982) qui, en analysant l'indifférence de Meursault avec les instruments de la critique sociologique et idéologique, le rapproche des personnages de Sartre, Moravia, Musil. Il peut ainsi faire apparaître le paradigme du héros indifférent dont l'importance culturelle et historique ne dépend pas seulement des vérités ou erreurs philosophiques de leurs auteurs ou de l'adéquation à des notions philosophiques. 
Certes, ce n'était pas là le projet de P.R. Et pourtant la lecture de son ouvrage nous porte à poser des questions fondamentales sur la « rentabilité » intellectuelle d'une approche si unilatérale, et en quelque sorte en circuit fermé, à une problématique qui est essentiellement bilatérale. D'ailleurs, l'auteur semble avoir une trace de mauvaise conscience, car il renvoie à plusieurs reprises à l'autre critique qu'on peut faire des œuvres littéraires et aux qualités intrinsèques du texte littéraire auxquelles se réfère cette critique. C'est une manière indirecte de tracer les contours de tout ce dont il a amputé une problématique frontalière qui connaît aujourd'hui d'intenses interactions entre philosophes et littéraires: les littéraires articulent des questions d'esthétique philosophique, des réflexions philosophiques sur l'écriture, alors que les philosophes s'intéressent de plus en plus aux questions de la mise en texte et de la matérialité discursive en les appliquant à leur propre activité. Même en philosophie les jours du mythe du langage comme transparence et comme véhicule neutre de l'Idée ou de la Vérité pure sont comptés. La critique philosophique du langage n'est pas passée inaperçue, un de ses résultats concrets nous montre que la philosophie est, elle aussi, un jeu de langage parmi d'autres. Aujourd'hui les «mystifications» et les «distorsions» par le langage ne constituent plus la differentia specifica de la littérature, ni le travail sur les concepts celle de la philosophie.

Programme de littérature comparée

Université de Montréal 